\title{
Characterisation of helium ion irradiated bulk tungsten: a comparison with the in-situ TEM technique
}

\author{
R.W. Harrison ${ }^{1}$, N. Peng ${ }^{2}$, R. P. Webb ${ }^{2}$, J.A. Hinks ${ }^{1}$ and S.E. Donnelly ${ }^{1}$ \\ ${ }^{1-}$ School of Computing and Engineering, University of Huddersfield, Huddersfield, HD1 \\ 3DH, UK \\ ${ }^{2-}$ Ion Beam Centre, University of Surrey, Guilford, GU2 7XH, UK
}

\begin{abstract}
Transmission electron microscopy (TEM) of ex-situ He ion irradiated bulk W has been performed to quantitatively compare the damage microstructure to that observed in regions of comparable thicknesses during in-situ ion irradiation with TEM experiments. Samples were irradiated to achieve He-appm/DPA ratios of 2,000 and 500 at temperatures of 500 and $800^{\circ} \mathrm{C}$ to 1.5 and $3.0 \mathrm{DPA}$. For irradiations at $500^{\circ} \mathrm{C}$, bubble diameters $(\sim 2 \mathrm{~nm})$ were larger and areal bubble densities $\left(\sim 10^{12}\right.$ bubbles $\left./ \mathrm{cm}^{2}\right)$ were lower than those in the in-situ experiments. This is attributed to greater amounts of He being retained in the ex-situ bulk experiments whereas in the in-situ experiments some may escape due to the proximity of surfaces. Dislocation loops were observed in all samples and were characterised as $\boldsymbol{b}= \pm 1 / 2<111>$ type with no $\boldsymbol{b}$ $=\langle 100\rangle$ type loops. Dislocation loop populations were dominated by interstitial type ( 60\%) agreeing with in-situ experiments. However, dislocation loops in this work were larger, ranging in size from $7-$ $100 \mathrm{~nm}$ and large concentrations of entangled dislocation lines were observed in the bulk of the grain as compared to in the in-situ experiments.
\end{abstract}

Keywords: Tungsten, Irradiation, Fusion, Radiation damage, TEM

\section{Introduction}

Tungsten is regarded as the primary candidate for use as a plasma facing material in the divertor of ITER and the Demonstration fusion power station (DEMO) due to its high melting temperature ( 3700 K), low sputter yield and high thermal conductivity ( $170 \mathrm{~W} / \mathrm{m} / \mathrm{K}$ at room temperature) [1]. During service, the divertor will be exposed to high heat fluxes, radiation damage from $14.1 \mathrm{MeV}$ neutrons and He injection from the plasma as well as He production from $(n, \alpha)$ reactions [2]. Production of He from $(\mathrm{n}, \alpha)$ reactions in $\mathrm{W}$ is low, with only around 5 atomic parts per million (appm) expected after two fullpower years in DEMO [3]. The $\alpha$ particles escaping from the plasma will generally be around $10 \mathrm{~s}$ of $\mathrm{eV}$, although particles with $\mathrm{MeV}$ energies have been measured escaping the plasma of the Joint European Torus (JET) reactor [4]. These particles will impact the $\mathrm{W}$ surface giving a range of $\mathrm{He}$ concentrations varying with depth and thus altering the He-appm to displacements per atom (DPA) ratio throughout the divertor structure. Hereafter, the He-appm/DPA ratio will be referred to as simply the He-appm/DPA ratio (in units of He appm per DPA). 
Given the anticipated He-appm/DPA ratios in the bulk of the divertor $(\sim 0)$ compared to the surface (>1000s due to He bombardment), it is critical to know how this will affect the performance of the W. Previous [5]-[7] in-situ irradiation studies, in which the He-appm/DPA ratio, temperature and total fluence (dose) were varied, explored the way in which these parameters affect the damage microstructure of W. However, an inherent aspect of in-situ transmission electron microscopy (TEM) techniques is the proximity of the surfaces given the requirement for thin electron-transparent samples. For example, Ferroni et al. [8] examined the effects of high temperature (up to $1400^{\circ} \mathrm{C}$ ) annealing of self-ion irradiated W noting that in-situ TEM experiments showed an accelerated loss of loops at $1000^{\circ} \mathrm{C}$ compared to ex-situ annealing experiments which may have been due to the greater proximity of surfaces in the TEM foils. The current work aims to compare the effects of the proximity of surfaces from our previous work using the TEM with in-situ ion irradiation technique [5], [6] with bulk ex-situ irradiations of comparable thicknesses at similar He-appm/DPA ratios, irradiation temperatures and DPAs.

\section{Experimental}

Samples of $\mathrm{W}$ were prepared by punching $3 \mathrm{~mm}$ discs from $0.1 \mathrm{~mm}$ thick foil (Alfa-Aesar, $99.95 \mathrm{wt} . \%$ ) main impurities as-received were $\mathrm{C} 0.005$ wt.\%, $\mathrm{Ca} 0.003$ wt.\% and $\mathrm{Al}, \mathrm{Cu}, \mathrm{Mg}, \mathrm{Si}, \mathrm{Ti}, \mathrm{Cr}, \mathrm{Mn}, \mathrm{Pb}$ and $\mathrm{Sn}<0.002 \mathrm{wt} . \%$. The discs were annealed at $1400^{\circ} \mathrm{C}$ for 2 hours under vacuum $(0.1 \mathrm{~Pa})$ to remove preexisting defects from cold working. The grain sizes were between $10-50 \mu \mathrm{m}$ as measured by focused ion beam-scanning electron microscope (FIB-SEM, FEI Quanta 3D).

Ex-situ ion irradiations were performed at the Ion Beam Centre (IBC) at the University of Surrey. Helium ion irradiations were performed with $400 \mathrm{keV}$ or $1.2 \mathrm{MeV}$ ions to achieve He-appm/DPA ratios of 2000 and $500 \mathrm{He}-\mathrm{appm} / \mathrm{DPA}$, respectively, at depths of $\sim 50-100 \mathrm{~nm}$ from the surface to match conditions from the in-situ irradiations. The incidence of the ion beam was normal to the sample surface and thus the same direction as the electron beam in post irradiation examination. The samples were irradiated at 500 and $800^{\circ} \mathrm{C}$ to 1.5 and 3.0 DPA. After irradiation, TEM samples were made by electropolishing the discs from the unirradiated side with $0.5 \mathrm{wt} . \% \mathrm{NaOH}$ solution at room temperature with a Tenupol-5. Samples were washed in three separate baths of $\mathrm{CH}_{3} \mathrm{OH}$ to remove any residue from the electrolyte. Figure 1a shows the DPA and He concentration (in appm) for $400 \mathrm{keV}$ at a fluence of 1 $\mathrm{x} 10^{18}$ ions $/ \mathrm{cm}^{2}$ and Figure $1 \mathrm{~b}$ shows the He-appm/DPA ratio at the foil depth analysed by TEM after electropolishing is $\sim 2000$. Figure $1 \mathrm{c}$ and d show the corresponding plots for $1.2 \mathrm{MeV} \mathrm{He}$ ions at a fluence of $2.8 \times 10^{18}$ ions $/ \mathrm{cm}^{2}$ showing the region analysed by TEM had a He-appm/DPA ratio $\sim 500$. Samples were irradiated at 500 and $800^{\circ} \mathrm{C}$ to doses of 1.5 and 3.0 DPA. Irradiation temperatures were chosen as to best match previous temperatures studied in our in-situ irradiations [5]-[7] and to best mimic the irradiation conditions relevant to fusion environments within the capability of the equipment. Damage and He concentrations were calculated using the Stopping and Range of Ions in Matter (SRIM- 
2013) Monte Carlo computer code [9]. DPA was calculated using the method proposed by Stoller et al. [10] using the 'Quick' Kinchin-Pease option of SRIM to 5000 ions with a displacement energy for W of $90 \mathrm{eV}$ [11] and lattice and surface binding energies set to $0 \mathrm{eV}$. Helium concentrations were determined from SRIM calculations using the 'Quick' method to 99,999 ions to ensure good statistics.

Bright-field (BF) micrographs of the irradiated samples were taken with a JEOL JEM-3010 TEM operated at $200 \mathrm{kV}$. Thickness measurements were made of both the in-situ and ex-situ irradiated TEM foils so that quantitative comparisons of the areal densities of defects could be made. Relative thicknesses were determined using a Gatan Model 666 parallel-detection electron energy-loss spectrometer (EELS). Regions analysed in the in-situ TEM foils were typically around 0.40-0.60 $\lambda$ and regions in the ex-situ foils were around $0.50-0.60 \lambda$ (where $\lambda$ is the inelastic mean free path for electrons and is $\sim 90 \mathrm{~nm}( \pm 20 \%)$ for $\mathrm{W}$ under the conditions used in this work). Helium bubbles were imaged using Fresnel contrast where small cavities appear light in underfocus and dark in overfocus. The image processing software ImageJ [12] was used to measure bubble size. Bubbles analysed were imaged under consistent underfocus conditions with a defocus value of $-1.0 \mu \mathrm{m}$ in order for quantitative comparisons to be made. The mean bubble sizes are reported as the averages of 30 measurements. Helium bubble densities were measured from TEM images by counting the number of bubbles in three regions of the same grain with the reported value given as the mean and the range as the standard deviation.

Analysis to determine the nature of dislocation loops was performed using the inside-outside contrast method [13], [14] on 20-30 loops. The loops analysed were at least $5 \mathrm{~nm}$ in size with $\boldsymbol{b}= \pm 1 / 2\langle 111\rangle$ and the sample was tilted about an electron beam direction of $z=[001]$. Excitation errors $\left(\boldsymbol{s}_{\mathrm{g}}\right)$ were set positive by tilting such that the Kikuchi line was on the opposite side of the corresponding diffraction spot with respect to the undiffracted beam [15]. In order to compare loops sizes between experiments, only loops with equivalent Burgers vectors imaged under the same two-beam diffraction conditions were compared. Dislocation loop size was measured as the major diameter of the core (i.e. the largest diameter of an oval shaped loop) as observed in projection. Areal dislocation loop densities were measured by superimposing images of the same regions taken under different two-beam diffraction conditions in order to include all loops present in the grain whilst also avoiding double counting.

\section{Results and Discussion}

\subsection{Helium bubble characterisation}

Helium bubbles were observed under all the irradiation conditions studied. Figure 2a-d show BF-TEM images of bubbles in $\mathrm{W}$ samples irradiated ex-situ with 1.2 MeV He ions (He-appm/DPA ratio 500). Figure $2 \mathrm{a}$ and $\mathrm{b}$ feature samples irradiated ex-situ to doses of 1.5 and 3.0 DPA, respectively, at a temperature of $500^{\circ} \mathrm{C}$ showing that the He bubble sizes and densities are similar for both. Figure $2 \mathrm{c}$ and $\mathrm{d}$ show samples irradiated ex-situ to 1.5 and 3.0 DPA, respectively, at a temperature of $800^{\circ} \mathrm{C}$, showing 
that He bubble size and density increased dramatically as a function of dose with the formation of large ( $>10 \mathrm{~nm}$ in diameter) faceted bubbles in the $800^{\circ} \mathrm{C}$ irradiation at 3.0 DPA. Figure 3a shows a plot of He bubble sizes as a function of DPA for both the 500 and $800^{\circ} \mathrm{C}$ ex-situ irradiations performed for the current work along with previous results from in-situ experiments [5], [7]. From this it can be seen that bubble sizes were around $2.0 \mathrm{~nm}$ and did not increase significantly between 1.5 and 3.0 DPA at an irradiation temperature of $500^{\circ} \mathrm{C}$ which agrees well with the in-situ experiments. From Figure $3 \mathrm{a}$ and $\mathrm{b}$ it can be seen that at an irradiation temperature of $500^{\circ} \mathrm{C}$, bubble diameters were larger, $>2 \mathrm{~nm}$ in the ex-situ irradiations compared to $\sim 1.5 \mathrm{~nm}$ in the in-situ irradiations although this falls within experimental errors. However, the areal bubble densities were significantly lower in the ex-situ ion irradiations as compared to in-situ. The larger He bubbles sizes with lower areal density observed in the ex-situ experiments may be attributed to lower fluxes of the ex-situ ion irradiations $\left(\sim 10^{12} \mathrm{ions} / \mathrm{cm}^{2} / \mathrm{s}\right.$ compared with $\sim 10^{13}$ ions $/ \mathrm{cm}^{2} / \mathrm{s}$ in the in-situ irradiations) allowing increased time for coalescence and growth of He bubbles.

Helium bubble diameters (Figure 3a) for the ex-situ irradiation temperature of $800^{\circ} \mathrm{C}$ increased from $\sim 3 \mathrm{~nm}$ at 1.5 DPA to $\sim 8 \mathrm{~nm}$ at 3.0 DPA which may be attributed to the high mobility of defects at the elevated temperature as compared to the irradiations at $500^{\circ} \mathrm{C}$. Defect mobility in $\mathrm{W}$ has been studied by electrical resistivity recovery mechanisms [8], [16]-[20]. Stage I recovery is attributed to the diffusion of free interstitials which are mobile at temperature below $-173^{\circ} \mathrm{C}$ [8]. Stage II recovery is defined as above $-173^{\circ} \mathrm{C}$ where interstitials are able to escape from traps and annihilate at sinks (e.g. immobile vacancies, grain boundaries or surfaces) [8]. Stage III is attributed to monovacancy migration in $\mathrm{W}$ and is associated with an activation energy of $1.7 \mathrm{eV}$ and becomes active at temperatures above $\sim 350^{\circ} \mathrm{C}$ [8], [21]. Stage IV occurs at temperatures above $\sim 720^{\circ} \mathrm{C}$ and is due to the mobility of vacancyimpurity complexes. Stage $\mathrm{V}$ (above $\sim 870^{\circ} \mathrm{C}$ ) is attributed to the migration of larger vacancy clusters [8].

Helium bubble diameter was not observed to increase significantly as a function of dose for an irradiation temperature of $500^{\circ} \mathrm{C}$, which is attributed to the irradiation temperature being just at or below the stage IV recovery step in W where vacancy-impurity complexes (i.e. small He-vacancy clusters) become mobile. The increase in size of the bubbles at the irradiation temperature of $800^{\circ} \mathrm{C}$ can be attributed to the increased mobility of monovacancies at $800^{\circ} \mathrm{C}$ and the activation of stage IV which occurs above $\sim 720^{\circ} \mathrm{C}$ due to the migration of vacancy-impurity complexes. The plot of bubble densities against irradiation temperature in Figure $3 \mathrm{~b}$ shows that at $800^{\circ} \mathrm{C}$ the ex-situ areal bubble density was initially similar to that at $750^{\circ} \mathrm{C}$ in the in-situ experiments at $1.5 \mathrm{DPA}$ from our previous work [5], [7]. However, as the dose is increased to 3.0 DPA the ex-situ density decreases and matches the areal density of bubbles observed in the in-situ experiments at $1000^{\circ} \mathrm{C}$. This decrease in areal bubble density as a function of dose may be attributed to the agglomeration of bubbles as they grow. Unfortunately, the insitu experiments at $1000^{\circ} \mathrm{C}$ were terminated at $\sim 2.0$ DPA due to intergranular fracture of the TEM foils. 
However, these bulk experiments suggest that the areal density would have decreased due to increased agglomeration.

Unlike in-situ experiments where bubble lattices formed under all $\mathrm{He}$-appm/DPA ratios at $500^{\circ} \mathrm{C}$ [6], random arrangements of bubbles were observed for the irradiation temperature of $500^{\circ} \mathrm{C}$ at both doses and He-appm/DPA ratios studied ex-situ. However, Figure 4 shows an area of bubble ordering in a W sample irradiated with a He-appm/DPA ratio $\sim 500$ at $500^{\circ} \mathrm{C}$ to 3.0 DPA. From Figure 4 it can be seen that bubbles are randomly distributed towards the centre of the grain. However, towards the grain boundary, ordering can be observed both in the image and via faint lines in the fast Fourier transform (FFT). However, this alignment appears to be along $\{200\}$ planes and is not along the $\{110\}$ planes along which bubble and void lattices are known to form in BCC metals [22]. Whereas helium bubble lattices have been observed in our previous in-situ work [6], the longer irradiation times of the ex-situ ion irradiation may have led to increased agglomeration of He bubbles leading to larger bubbles forming and destroying any bubble ordering.

\subsection{Dislocation loop characterisation}

Dislocation loops were observed for both He-appm/DPA ratios studied in the ex-situ irradiations at irradiation temperatures of $500^{\circ} \mathrm{C}$ and $800^{\circ} \mathrm{C}$. From Figure 5 it can be seen that at lower doses and lower irradiation temperatures the dislocation populations consisted of a mixture of line and loop dislocations for both He-appm/DPA ratios. However, at higher doses and temperatures the dislocation population appears to be dominated by entangled dislocation loops and lines. Due to the large concentrations of dislocation loops and lines in the bulk of the grains, dislocation imaging and analysis could only be performed on samples with lower density of dislocations and at grain boundaries where their concentrations were lower so as to get good resolution of their contrast.

Using the $\boldsymbol{g} . \boldsymbol{b}=0$ criterion, the loops were characterised as $\boldsymbol{b}= \pm 1 / 2<111\rangle$ type with no $\langle 100\rangle$ type observed. Figure 6 shows an example of Burgers vector analysis for a sample irradiated at $500^{\circ} \mathrm{C}$ using 1.2 MeV He ions to 3.0 DPA imaged close to the $z=[001]$ zone axis. It can be seen that the dislocation loops marked with squares are visible in Figure $6 \mathrm{a}$, b, e and f where $g=020,0 \overline{2} 0, \overline{1} 10$ and $1 \overline{1} 0$, respectively, and that they are invisible in Figure $6 \mathrm{c}$ and d where $g=\overline{1} \overline{1} 0$ and 110 , respectively. The loops marked with squares also showed no contrast when $g=\overline{2} \overline{1} 1$ imaged close to $z=$ [113] which is consistent with $\boldsymbol{b}= \pm 1 / 2[1 \overline{1} 1]$. The observation of only $\boldsymbol{b}= \pm 1 / 2<111>$ is contrary to previous work on self-ion irradiation reported in the literature [27]. However, it is in agreement with the in-situ He ion irradiations that we previously reported [5], [7].

In BCC structures, <100> type loops have been proposed to form via two mechanisms. Eyre and Bullough [28] reported that faulted loops nucleate on $\{110\}$ planes which then shear as they grow, due to their containing a stacking fault, into either $\boldsymbol{b}= \pm 1 / 2<111>$ or $<100>$ type loops with the un-faulting 
into the $\boldsymbol{b}= \pm 1 / 2<111>$ being much more favourable [29]. However, the formation of $<100>$ loops via the reaction of two $\pm 1 / 2<111>$ loops has was proposed by Masters [30] and this has been shown in $\mathrm{FeCr}$ alloys experimentally [31], [32]. Under dual self-ion and He irradiations, damage microstructures of $\mathrm{FeCr}$ were found to be dominated by $\boldsymbol{b}= \pm 1 / 2<111>$ loops as compared to the single self-ion irradiation case which was a mixture of $\boldsymbol{b}= \pm 1 / 2<111>$ and $<100>$ type loops [31], [32]. This is attributed to He decreasing the mobility of $\pm 1 / 2<111>$ type loops, leading to lower probability of interaction and coalescence with another $\pm 1 / 2<111>$ type loop [31]. Recent MD simulations by Sand et al. [33] found that the formation of $\boldsymbol{b}=\langle 100\rangle$ loops occurred as a direct result of the cascade collapse, agreeing well with the work of Yi et al. [34], [35]. This is attributed to the large energy density of cascades giving rise to longer lived thermal spikes and higher chance of loops un-faulting into $\boldsymbol{b}=\langle 100\rangle$ type [36]. Helium ions will produce widely spaced, dilute cascades formed with many isolated Frenkel pairs [37] and a very low energy density as compared with self-ions. We have previously shown [38] that He has no effect on the type of dislocation loop formed in self-ion irradiated $\mathrm{W}$, unlike in the $\mathrm{FeCr}$ alloys [31], [32] and that $\boldsymbol{b}=\langle 100\rangle$ type loops arise in the presence of dense cascade damage. Thus, the lack of $\boldsymbol{b}$ $=\langle 100\rangle$ in this work is attributed to the lack of dense cascade damage.

The nature of the loops (i.e. vacancy or interstitial) for the samples irradiated to a He-appm/DPA ratio of 500 to a dose of $3.0 \mathrm{DPA}$ at $500^{\circ} \mathrm{C}$ was characterised using the inside-outside method; an example of which is shown in Figure 6. The loops in the solid squares were characterised as $\boldsymbol{b}= \pm 1 / 2[1 \overline{1} 1]$ using the $\boldsymbol{g} \cdot \boldsymbol{b}=0$ criterion. It can be seen that these loops show outside contrast in Figure $6 \mathrm{a}$ and e where $\boldsymbol{g}=$ 020 and $\overline{1} 10$, respectively, with $\boldsymbol{s}_{\mathrm{g}}>0$ leading to the loop inclination being determined to be $\boldsymbol{b}=-1 / 2[1 \overline{1} 1]$ (for outside contrast $(\boldsymbol{g} . \boldsymbol{b}) \boldsymbol{s}_{\mathrm{g}}>0$ ). As $z=[001], \boldsymbol{b} . z<0$ and therefore these loops are interstitial in nature [13], [14], [39]. Dashed circles in Figure 6 show vacancy-type loops with $\boldsymbol{b}=+1 / 2[111]$ and the solid circles indicate interstitial loops with $\boldsymbol{b}=-1 / 2[111]$. The formation of vacancy type-loops in the He ion irradiation case [5] is an intriguing one, and would not appear to be compatible with a nucleation mechanism from cascade collapse as in the Au [40] and W ion [27], [34], [35] irradiations in which vacancy-type loops can nucleate via cascade collapse. As the irradiation temperatures in the He ion irradiation case [5] were all at or above $500^{\circ} \mathrm{C}$ this will be above the Stage III recovery stage in W where monovacancies are mobile [8], [21]. Thus, in the absence of dense cascade damage (as in the case of our He ion irradiation) vacancy-type loops are likely to nucleate via vacancies accumulating and coalescing by thermal migration and forming extended defect clusters which then collapse into dislocation loops. From Figure 6 it can be seen that at $3.0 \mathrm{DPA}$ at $500^{\circ} \mathrm{C}$ some vacancy loops were of the order of $\sim 100 \mathrm{~nm}$ in diameter. As their suspected mechanism for nucleation and growth requires the mobility of monovacancies it seems surprising that loops of this size may form at $500^{\circ} \mathrm{C}$, which is only just above the monovacancy migration temperature of $\sim 350^{\circ}$ (Stage III recovery). Ferroni et al. [8] noted the formation of large $(>100 \mathrm{~nm})$ dislocation loops in self-ion irradiated $\mathrm{W}$ annealed to $800^{\circ} \mathrm{C}$. The loops were predominantly interstitial in nature with $\sim 20 \%$ being vacancy type, however, the authors 
do not discriminate between the sizes of the interstitial and vacancy type loops. Rau et al. [41] annealed neutron irradiated $\mathrm{W}$ to $1100^{\circ} \mathrm{C}$ and reported the presence of vacancy dislocation loops with $\boldsymbol{b}=$ $\pm 1 / 2<111>$ type only which had an average diameter of $\sim 100 \mathrm{~nm}$. Although these dislocation sizes seem to match well with the loop sizes in Figure 6, samples were annealed to higher temperatures than the irradiation temperature in this work. Although the irradiation temperature in this work is just above the vacancy diffusion temperature, the production of dilute cascades consisting mainly of isolated Frenkel pairs may enhance their diffusivity dramatically enough to grow to the sizes comparable with higher temperature anneals over the longer timescales of the ex-situ ion irradiations. The relative populations of interstitial- and vacancy-type loops is $\sim 60: 40$, agreeing well with the TEM with in-situ ion irradiation experiments [5] at $500^{\circ} \mathrm{C}$. This relatively-higher number of interstitial-type loops compared to vacancytype is attributed to the stronger bias of dislocations for interstitial absorption whereas other sinks such as grain boundaries and cavities are neutral [37]. Helium bubbles will also act as trapping sites for vacancies reducing the number in flux leading to fewer vacancy type loops growing to visible sizes in the TEM.

Compared with the in-situ experiments however, dislocation loop sizes were much larger and areal dislocation densities were lower in this ex-situ study. For example, in the in-situ irradiations, dislocation loop sizes at 3.0 DPA at $500^{\circ} \mathrm{C}$ were $\sim 5-15 \mathrm{~nm}$ for a He-appm/DPA of $\sim 500$ and had an areal density of $\sim 10^{11}$ dislocations $/ \mathrm{cm}^{2}$. However in the ex-situ experiments, dislocation loops of the same type (i.e. interstitial) and Burgers vector imaged under the same diffraction conditions (to allow valid comparison) showed a much larger range of sizes from $\sim 7-100 \mathrm{~nm}$ and a much lower areal density of $\sim 10^{10}$ dislocations $/ \mathrm{cm}^{2}$. In addition, there was a change in the dislocation microstructure from the in-situ experiments, which predominantly consisted of isolated loops compared to larger more-complex networks of dislocation loops and lines in the ex-situ experiments. This difference may be attributed to the nature of the experiments with the surfaces in the in-situ irradiations providing large sinks for the annihilation of migrating point defects, small clusters and mobile dislocations. This will lead to a higher density of small, immobile clusters or dislocation loops that grow much more slowly as there will be a lower point-defect flux due to the increased annihilation at the surfaces. However, in the ex-situ experiments where these surface effects are likely to be significantly lower than in the in-situ work, dislocation networks will grow at a much faster rate, become larger and lead to the dislocation networks observed.

\section{Conclusions}

Bulk samples of W have been irradiated ex-situ with $\mathrm{He}$ ions at $400 \mathrm{keV}$ and $1.2 \mathrm{MeV}$ to achieve $\mathrm{He}$ appm/DPA ratios of 2000 and 500, respectively, at temperatures of 500 and $800^{\circ} \mathrm{C}$ up to a maximum dose of 3.0 DPA. These conditions were chosen to match in-situ irradiations performed in a TEM [5][7] to compare the damage microstructures from the two sets of experiments. Bubble diameters were 
larger and areal bubble density was lower in the ex-situ, compared to the in-situ, experiments. Unlike in-situ TEM experiments, random arrangements of bubbles were observed for the irradiation temperature of $500^{\circ} \mathrm{C}$ and all doses at both He-appm/DPA ratios studied ex-situ. Dislocation loops were characterised as $\boldsymbol{b}= \pm 1 / 2<111\rangle$ type with no $\boldsymbol{b}=\langle 100\rangle$ type loops detected due to the absence of dense cascade damage. Dislocation loops in this work were larger than in the TEM with in-situ ion irradiation experiments and ranged in size from $\sim 7-100 \mathrm{~nm}$ with a lower areal density of $\sim 10^{10}$ dislocations $/ \mathrm{cm}^{2}$. A mixture of entangled dislocation loops and lines have been observed in the ex-situ bulk irradiation experiments unlike the in-situ TEM experiments.

The differences in the defect microstructures in the in-situ and ex-situ cases may be attributed to larger concentrations of $\mathrm{He}$ and defect clusters being retained in the ex-situ bulk experiments leading to larger defects (He bubbles and dislocations); these may annihilate at the TEM foil surface in the in-situ case. In addition, the lower flux of the ex-situ irradiations will lead to increased coalesce and growth of defects resulting in fewer, larger defects observed in this work.

\section{Acknowledgments}

The authors are grateful to the EPSRC for financial support of this project through grants EP/M011135/1 and funding for UK National Ion Beam Centre as a Mid-Range Facility

\section{Declaration of interests}

No potential conflict of interest was reported by the authors 


\section{References}

[1] H. Bolt, V. Barabash, W. Krauss, J. Linke, R. Neu, S. Suzuki, N. Yoshida, and A. U. Team, Materials for the plasma-facing components of fusion reactors, vol. 329-333, no. 1-3 PART A. 2004, pp. 66-73.

[2] J.-C. Sublet, J. W. Eastwood, and J. G. Morgan, "EASY-II Renaissance: n, p, d, $\alpha, \gamma$-induced Inventory Code System,” Nucl. Data Sheets, vol. 118, pp. 115-117, 2014.

[3] M. R. Gilbert, S. L. Dudarev, S. Zheng, L. W. Packer, and J.-C. Sublet, "An integrated model for materials in a fusion power plant: transmutation, gas production, and helium embrittlement under neutron irradiation," Nucl. Fusion, vol. 52, no. 8, p. 083019, 2012.

[4] D. S. Darrow, F. E. Cecil, V. Kiptily, K. Fullard, A. Horton, and A. Murari, "Observation of alpha particle loss from JET plasmas during ion cyclotron resonance frequency heating using a thin foil Faraday cup detector array," Rev. Sci. Instrum., vol. 81, no. 10, p. 10D330, Oct. 2010.

[5] R. W. Harrison, G. Greaves, J. A. Hinks, and S. E. Donnelly, "A study of the effect of helium concentration and displacement damage on the microstructure of tungsten irradiation with helium ions," J. Nucl. Mater., vol. 495, pp. 492-503, 2017.

[6] R. W. Harrison, G. Greaves, J. A. Hinks, and S. E. Donnelly, "Engineering self-organising helium bubble lattices in tungsten," Sci. Rep., vol. 7, p. 7724, 2017.

[7] R. W. Harrison, H. Amari, G. Greaves, J. A. Hinks, and S. E. Donnelly, "Effect of Heappm/DPA ratio on the damage microstructure of tungsten," MRS Adv., vol. 1, no. 42, pp. 2893-2899, 2016.

[8] F. Ferroni, X. Yi, K. Arakawa, S. P. Fitzgerald, P. D. Edmondson, and S. G. Roberts, "High temperature annealing of ion irradiated tungsten," Acta Mater., vol. 90, pp. 380-393, 2015.

[9] J. F. Ziegler, "Stopping of energetic light ions in elemental matter," J. Appled Phys., vol. 85, no. 3, p. 1249, 1999.

[10] R. E. Stoller, M. B. Toloczko, G. S. Was, A. G. Certain, S. Dwaraknath, and F. A. Garner, "On the use of SRIM for computing radiation damage exposure," Nucl. Instruments Methods Phys. Res. Sect. B Beam Interact. with Mater. Atoms, vol. 310, pp. 75-80, 2013.

[11] A. E521, "Standard Practice for Neutron Radiation Damage Simulation by Charged-Particle," Annu. B. ASTM Stand., vol. 12.02, no. Reapproved, pp. 1-21, 2009.

[12] C. A. Schneider, W. S. Rasband, and K. W. Eliceiri, "NIH Image to ImageJ: 25 years of image analysis," Nat Meth, vol. 9, no. 7, pp. 671-675, Jul. 2012.

[13] D. M. Maher and B. L. Eyre, "Neutron irradiation damage in molybdenum," Philos. Mag., vol. 23, no. 182 , pp. 409-438, 1971.

[14] M. L. Jenkins, "Characterisation of radiation-damage microstructures by TEM," J. Nucl. Mater., vol. 216, no. C, pp. 124-156, 1994.

[15] D. B. Williams and C. B. Carter, "Electron Energy-Loss Spectrometers and Filters," in Transmission Electron Microscopy: A Textbook for Materials Science, Boston, MA, MA: Springer US, 2009, pp. 679-698. 
[16] A. Hasegawa, M. Fukuda, S. Nogami, and K. Yabuuchi, "Neutron irradiation effects on tungsten materials," Fusion Eng. Des., vol. 89, pp. 1568-1572, 2014.

[17] F. Maury, M. Biget, P. Vajda, A. Lucasson, and P. Lucasson, "Frenkel pair creation and stage I recovery in W crystals irradiated near threshold," Radiat. Eff. Inc. Plasma Sci. Plasma Technol., vol. 38, pp. 1-2, 1978.

[18] T. Tanno, a. Hasegawa, J. C. He, M. Fujiwara, M. Satou, S. Nogami, K. Abe, and T. Shishido, "Effects of transmutation elements on the microstructural evolution and electrical resistivity of neutron-irradiated tungsten," J. Nucl. Mater., vol. 386-388, no. 2009, pp. 218-221, 2009.

[19] R. W. Balluffi, "Vacancy defect mobilities and binding energies obtained from annealing studies," J. Nucl. Mater., vol. 69-70, pp. 240-263, 1978.

[20] D. R. Mason, X. Yi, M. A. Kirk, and S. L. Dudarev, "Elastic trapping of dislocation loops in cascades in ion-irradiated tungsten foils," J. Phys. Condens. Matter, vol. 26, no. 37, pp. 1-28, 2014.

[21] O. El-Atwani, J. A. Hinks, G. Greaves, S. Gonderman, T. Qiu, M. Efe, and J. P. Allain, "Insitu TEM observation of the response of ultrafine- and nanocrystalline-grained tungsten to extreme irradiation environments," Sci. Rep., vol. 4, no. 1, p. 4716, 2015.

[22] P. B. P. Johnson and D. J. D. Mazey, "Gas-bubble superlattice formation in bcc metals," $J$. Nucl. Mater., vol. 218, no. 3, pp. 273-288, Mar. 1995.

[23] P. B. Johnson and D. J. Mazey, "Gas-bubble superlattice formation in bcc metals," J. Nucl. Mater., vol. 218, no. 3, pp. 273-288, Mar. 1995.

[24] J. H. Evans, "Irradiation-Induced Cavity Lattice Formation in Metals," in Patterns, Defects and Materials Instabilities, D. Walgraef and N. M. Ghoniem, Eds. Dordrecht: Springer Netherlands, 1990, pp. 347-370.

[25] K. Krishan, "Invited review article ordering of voids and gas bubbles in radiation environments," Radiat. Eff., vol. 66, no. 3-4, pp. 121-155, 1982.

[26] V. K. Sikka and J. Moteff, "Superlattice of voids in neutron-irradiated tungsten," J. Appl. Phys., vol. 43, no. 12, pp. 4942-4944, 1972.

[27] X. Yi, M. L. Jenkins, K. Hattar, P. D. Edmondson, and S. G. Roberts, "Characterisation of radiation damage in $\mathrm{W}$ and $\mathrm{W}$-based alloys from $2 \mathrm{MeV}$ self-ion near-bulk implantations," Acta Mater., vol. 92, pp. 163-177, 2015.

[28] B. L. Eyre and R. Bullough, "On the formation of interstitial loops in b.c.c. metals," Philos. Mag. A J. Theor. Exp. Appl. Phys., vol. 12, no. 115, pp. 31-39, Jul. 1965.

[29] C. A. English and M. L. Jenkins, "Molecular ion irradiations of molybdenum," Philos. Mag., vol. 90, no. 7-8, pp. 821-843, 2010.

[30] B. C. Masters, "Dislocation loops in irradiated iron," Philos. Mag. A J. Theor. Exp. Appl. Phys., vol. 11, no. 113, pp. 881-893, 1965. 
[31] A. Prokhodtseva, B. Décamps, A. Ramar, and R. Schäublin, "Impact of He and Cr on defect accumulation in ion-irradiated ultrahigh-purity Fe(Cr) alloys," Acta Mater., vol. 61, no. 18, pp. 6958-6971, 2013.

[32] R. Schäublin, B. Décamps, A. Prokhodtseva, and J. F. Löffler, "On the origin of the primary $1 / 2$ $\mathrm{a} 0<111>$ and $\mathrm{a} 0<100>$ loops in irradiated $\mathrm{Fe}(\mathrm{Cr})$ alloys," Acta Mater., vol. 133, pp. 427-439, 2017.

[33] A. E. Sand, S. L. Dudarev, and K. Nordlund, "High-energy collision cascades in tungsten: Dislocation loops structure and clustering scaling laws," EPL (Europhysics Lett., vol. 103, no. 4, p. 46003, 2013.

[34] X. Yi, M. L. Jenkins, M. Briceno, S. G. Roberts, and Z. Zhou, "In situ study of self-ion irradiation damage in $\mathrm{W}$ and $\mathrm{W}-5 \mathrm{Re}$ at $500^{\circ}$ C," Philos. Mag. A, vol. 93, no. July 2015, pp. 1715-1738, 2013.

[35] X. Yi, M. L. Jenkins, M. A. Kirk, Z. Zhou, and S. G. Roberts, "In-situ TEM studies of 150 $\mathrm{keV} \mathrm{W}+$ ion irradiated W and W-alloys: Damage production and microstructural evolution," Acta Mater., vol. 112, pp. 105-120, 2016.

[36] C. A. English and M. L. Jenkins, "Insight into Cascade Processes Arising from Studies of Cascade Collapse," Mater. Sci. Forum, vol. 15-18, pp. 1003-1022, 1987.

[37] G. S. Was, Fundamentals of Radiation Materials Science, 1st ed. New York: Springer-Verlag Berlin Heidelberg, 2007.

[38] R. W. Harrison, J. A. Hinks, and S. E. Donnelly, "Influence of pre-implanted helium on dislocation loop type in tungsten under self-ion irradiation," Scr. Mater., vol. 150, no. C, pp. 61-65, 2018.

[39] H. Föll and M. Wilkens, "A simple method for the analysis of dislocation loops by means of the inside-outside contrast on transmission electron micrographs," Phys. Status Solidi, vol. 31, no. 2, pp. 519-524, Oct. 1975.

[40] W. Jäger and M. Wilkens, "Formation of vacancy-type dislocation loops in tungsten bombarded by $60 \mathrm{keV}$ Au ions," Phys. status solidi, vol. 32, no. 1, pp. 89-100, Nov. 1975.

[41] R. C. Rau, "Vacancy dislocation loops in irradiated and annealed tungsten," Philos. Mag. A J. Theor. Exp. Appl. Phys., vol. 18, no. 155, pp. 1079-1084, 1968. 


\section{List of figures}

Figure 1. a) DPA and He implantation plot as a function of depth for $400 \mathrm{keV} \mathrm{He}$ ions at a fluence of $10^{18}$ ions $/ \mathrm{cm}^{2}$; b) He-appm/DPA plot as a function of depth from area marked in (a) showing the ratio in the section of the ex-situ irradiated bulk sample analysed in TEM is 2000; c) DPA and He implantation plots as a function of depth for $1.2 \mathrm{MeV}$ He ions for a fluence of $2.8 \times 10^{18}$ ions $/ \mathrm{cm}^{2}$; and d) He-appm/DPA plot as a function of depth from area marked in (c) showing the ratio in the TEM analysed section from electropolishing is $\sim 500$

Figure 2. BF-TEM images taken at $-1.0 \mu \mathrm{m}$ underfocus of bulk $\mathrm{W}$ irradiated samples irradiated ex-situ with $1.2 \mathrm{MeV} \mathrm{He}$ ions to achieve a He-appm/DPA ratio of 500 at: a) $500^{\circ} \mathrm{C}$ and $1.5 \mathrm{DPA}$ with white box highlighting area enlarged to show bubbles of around $2 \mathrm{~nm}$ in size; b) $500^{\circ} \mathrm{C}$, and $3.0 \mathrm{DPA}$ with white box highlighting area to show bubbles are also around $2 \mathrm{~nm}$; c) $800^{\circ} \mathrm{C}$ and $1.5 \mathrm{DPA}$ with white box highlighting area enlarged to show bubbles of around $3 \mathrm{~nm}$ in size; and d) $800^{\circ} \mathrm{C}$ and $3.0 \mathrm{DPA}$ with white box highlighting area enlarged to show bubbles of around $8 \mathrm{~nm}$ in size. The scale bar applies to all micrographs in figure.

Figure 3. Comparison of bulk W samples irradiated ex-situ with 1.2 MeV He ions (He-appm/DPA 500) compared with the in-situ results from our previous works [5]: a) bubble diameter as a function of DPA; and b) areal bubble densities as a function of DPA. .15

Figure 4. BF-TEM image at $-1.0 \mu \mathrm{m}$ underfocus of a bulk W sample ex-situ irradiated with $1.2 \mathrm{MeV}$ $\mathrm{He}$ ions (He-appm/DPA $\sim 500)$ at $500^{\circ} \mathrm{C}$ to $3.0 \mathrm{DPA}$ showing a random bubble distribution in the majority of the grain but bubble ordering closer to the grain boundary (dashed white box). The FFT of the area inside the dashed box shows ordering in $2 \mathrm{D}$.

Figure 5. BF-TEM images of bulk W ex-situ irradiated samples imaged under two-beam conditions with $g=(200)$ : The scale bar in lower left image applies to all micrographs in figure.

Figure 6. Two-beam TEM images (taken close to focus) of bulk W sample irradiated ex-situ at $500^{\circ} \mathrm{C}$ with 1.2 $\mathrm{MeV} \mathrm{He}^{+}$ions to achieve an He-appm/DPA ratio of 500 to $3.0 \mathrm{DPA}$ : a) $\boldsymbol{g}=020$; b) $\boldsymbol{g}=020$; c) $\boldsymbol{g}=110$; d) $\boldsymbol{g}=110$; e) $\boldsymbol{g}=110$; and f) $\boldsymbol{g}=110$. Solid squares indicate vacancy loops with $\boldsymbol{b}=1 / 2[111]$, solid circles indicate interstitial loops with $\boldsymbol{b}=1 / 2[111]$ and dashed circles indicate vacancy loops with $\boldsymbol{b}=1 / 2[111]$. The scale bar applies to all micrographs in figure .18 
a)

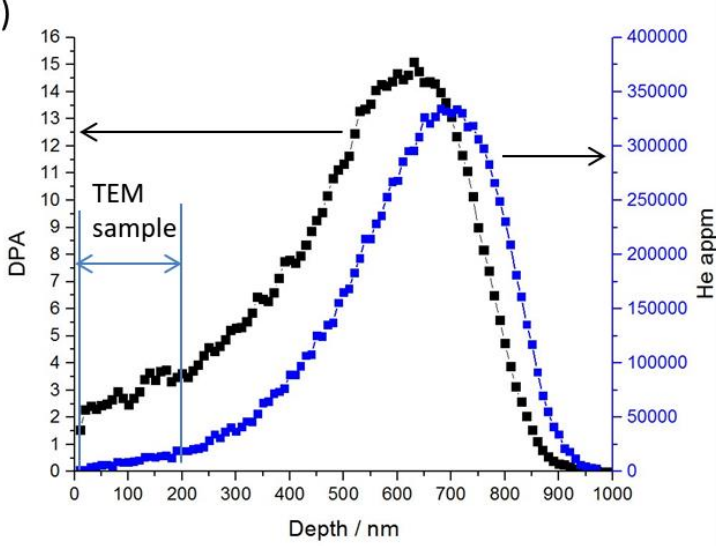

c)

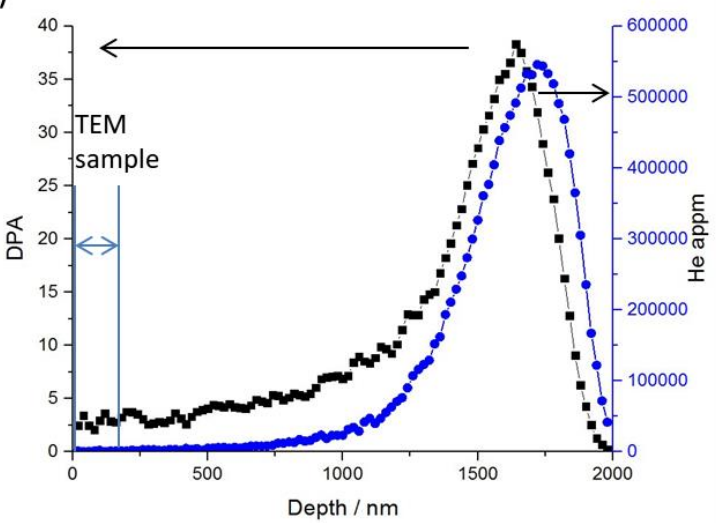

b)

TEM sample Polishing direction

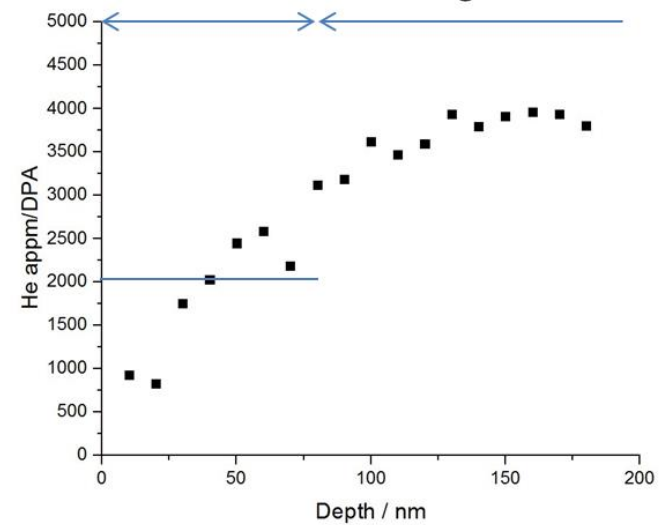

d)

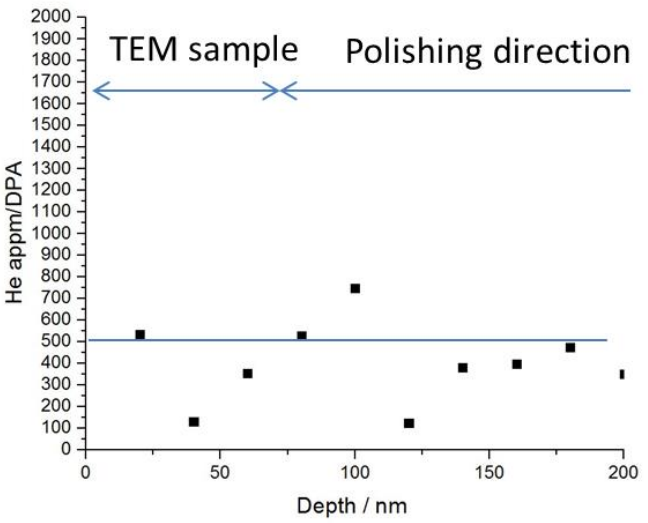

Figure 1. a) DPA and He implantation plot as a function of depth for $400 \mathrm{keV} \mathrm{He}$ ions at a fluence of $10^{18}$ ions $/ \mathrm{cm}^{2}$; b) He-appm/DPA plot as a function of depth from area marked in (a), c) DPA and He implantation plots as a function of depth for $1.2 \mathrm{MeV} H e$ ions and d) He-appm/DPA plot as a function of depth from area marked in (c) 

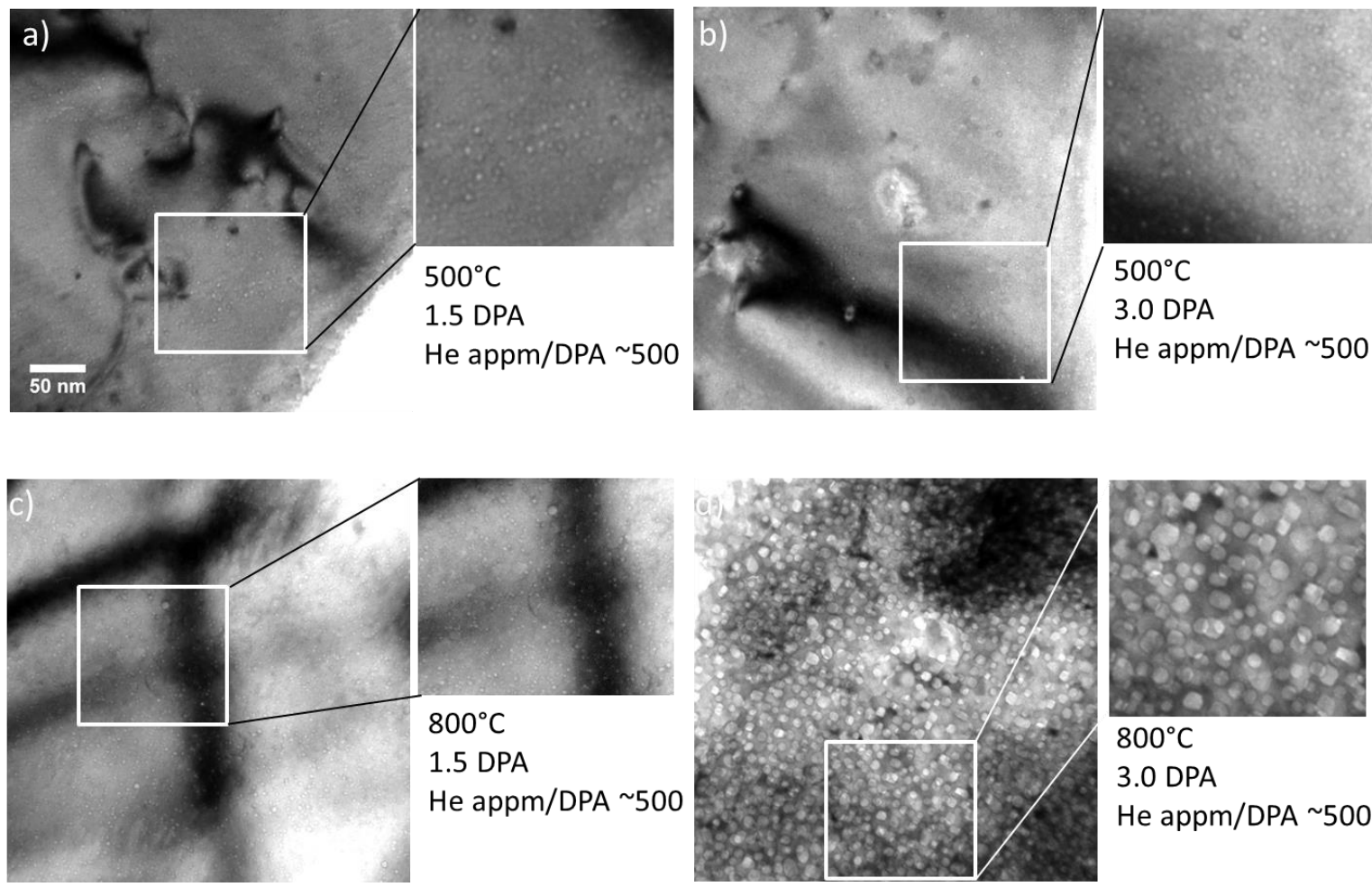

$800^{\circ} \mathrm{C}$

3.0 DPA

He appm/DPA $\sim 500$

Figure 2. BF-TEM images taken at $-1.0 \mu \mathrm{m}$ underfocus of $\mathrm{W}$ samples irradiated with $1.2 \mathrm{MeV} \mathrm{He}$ ions at different temperatures and DPAs (scale bar applies to all micrographs in figure). 
a)

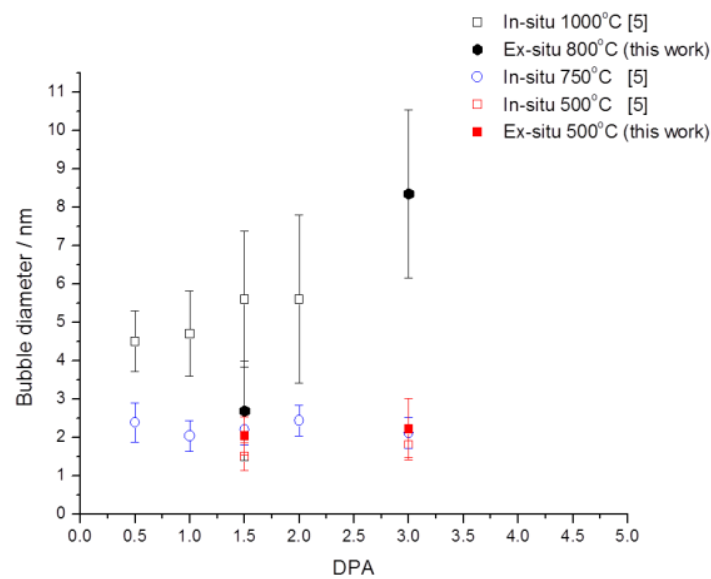

b)

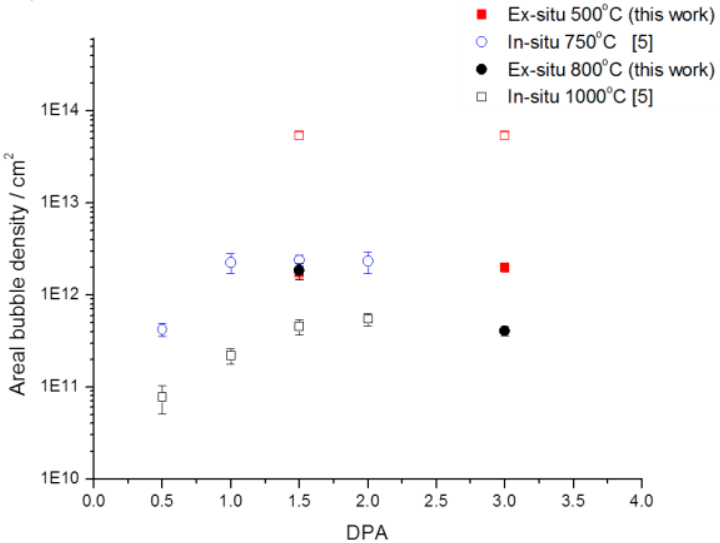

Figure 3. Comparison of bulk W samples irradiated ex-situ with $1.2 \mathrm{MeV} \mathrm{He}$ ions (He-appm/DPA 500) compared with the in-situ results from our previous works [5]: a) bubble diameter as a function of DPA; and b) areal bubble densities as a function of DPA. 


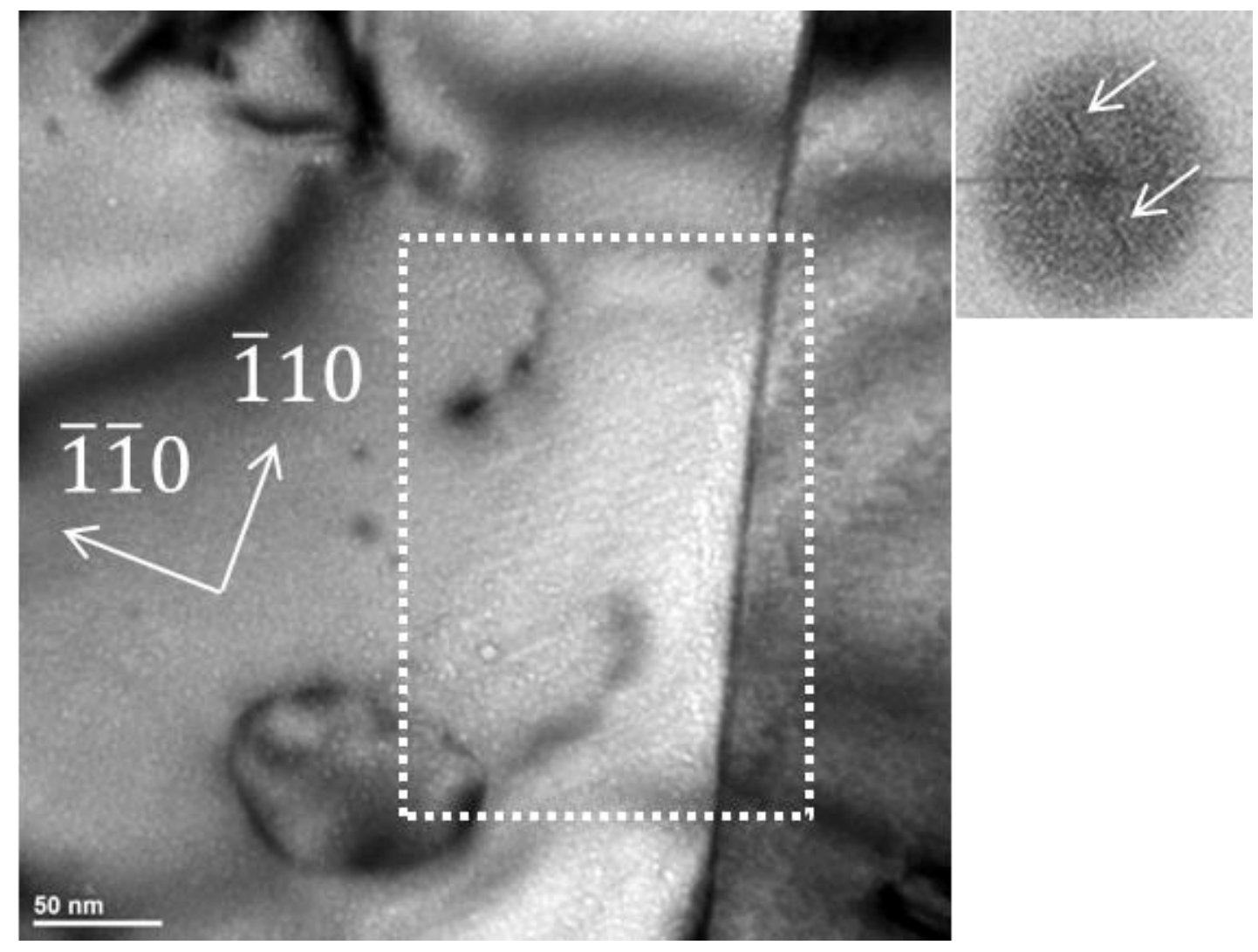

Figure 4. BF-TEM image at $-1.0 \mu \mathrm{m}$ underfocus of a bulk W sample ex-situ irradiated with $1.2 \mathrm{MeV}$ He ions (He-appm/DPA 500) at $500^{\circ} \mathrm{C}$ to 3.0 DPA showing a random bubble distribution in the majority of the grain but bubble ordering closer to the grain boundary (dashed white box). The FFT of the area inside the dashed box shows ordering in $2 \mathrm{D}$. 


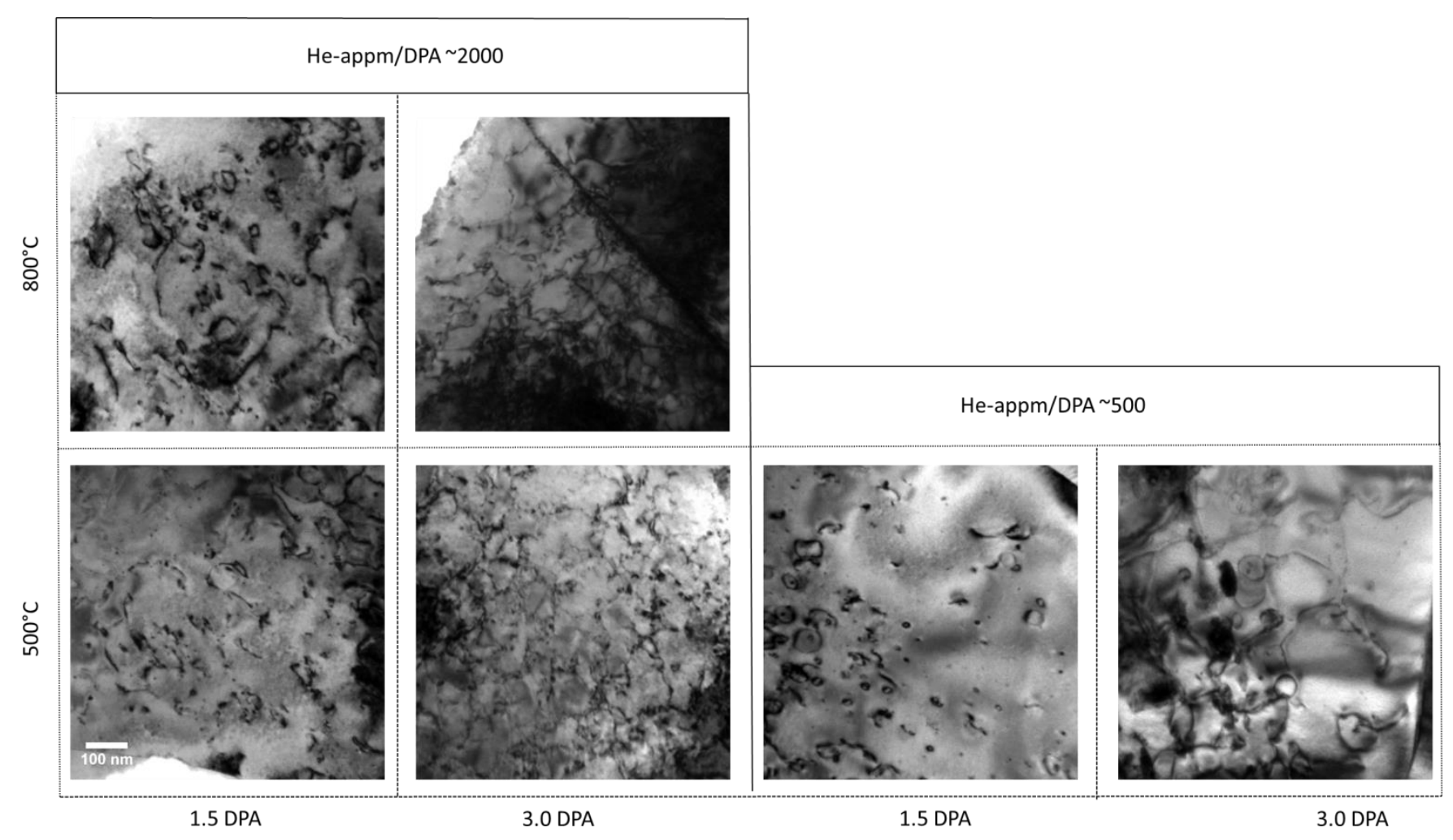

Figure 5. BF-TEM images of bulk W ex-situ irradiated samples imaged under two-beam conditions with $g=(200)$ : The scale bar in lower left image applies to all micrographs in figure. 

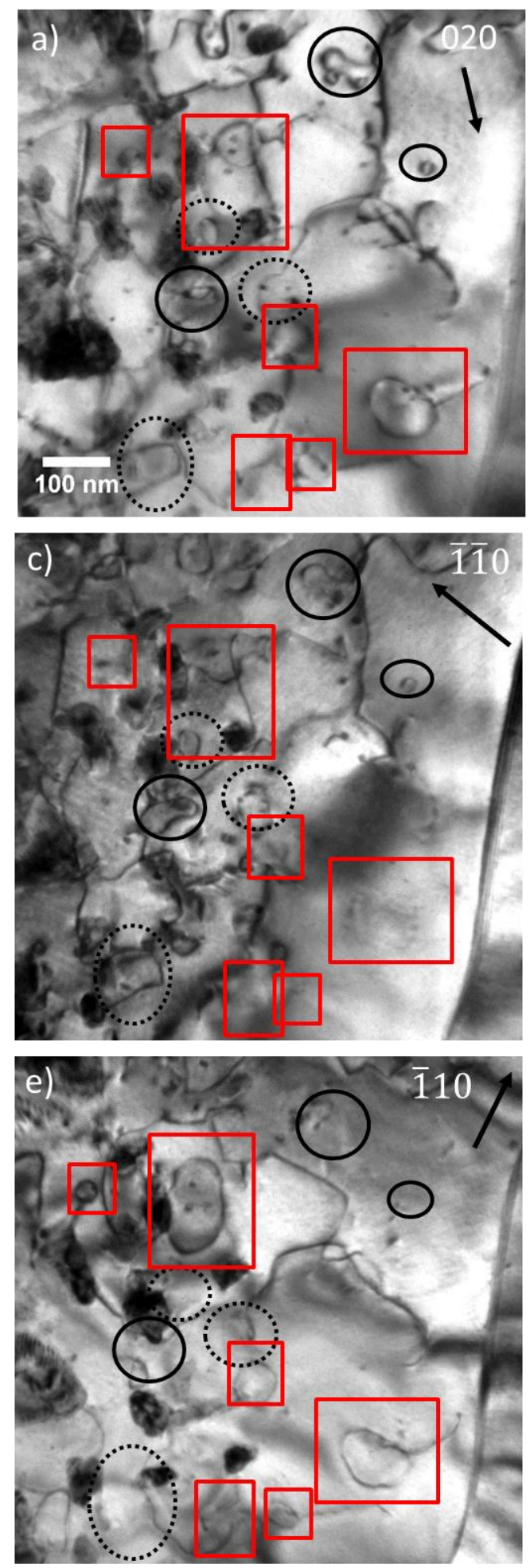
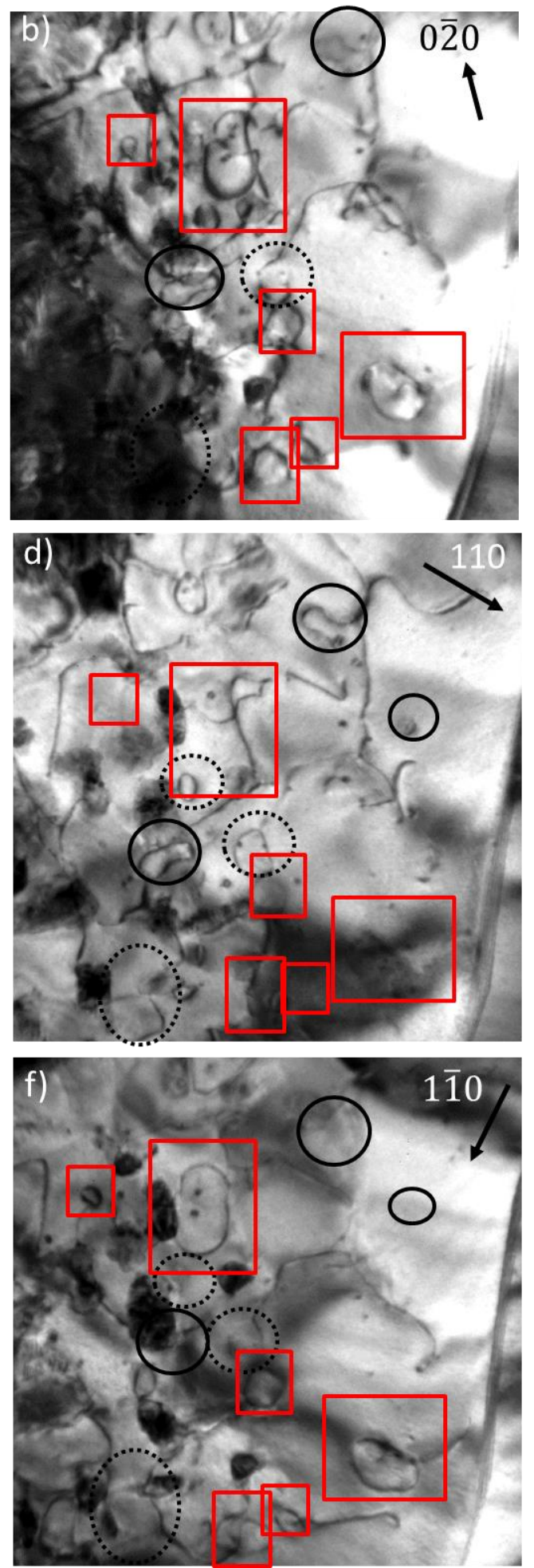

Figure 6. Two-beam TEM images (taken close to focus) of bulk W sample irradiated ex-situ at $500^{\circ} \mathrm{C}$ with 1.2 $\mathrm{MeV} \mathrm{He}^{+}$ions to achieve an He-appm/DPA ratio of 500 to $3.0 \mathrm{DPA}$ : a) $\boldsymbol{g}=020$; b) $\boldsymbol{g}=0 \overline{2} 0$; c) $\boldsymbol{g}=\overline{1} \overline{1} 0$; d) $\boldsymbol{g}=110$; e) $\boldsymbol{g}=\overline{1} 10$; and f) $\boldsymbol{g}=1 \overline{1} 0$. Solid squares indicate interstitial loops with $\boldsymbol{b}=$ $1 / 2[1 \overline{1} 1]$, solid circles indicate interstitial loops with $\boldsymbol{b}=1 / 2[111]$ and dashed circles indicate vacancy loops with $\boldsymbol{b}=1 / 2[111]$. The scale bar applies to all micrographs in figure. 